\title{
Existence Results of Three-Point Boundary Value Problems for Second-order Ordinary Differential Equations
}

\author{
Sheng-Ping Wang ${ }^{1}$ and Long-Yi Tsai ${ }^{2}$ \\ ${ }^{1}$ Holistic Education Center, Cardinal Tien College of Healthcare and Management, No.11, Zhongxing Road, \\ Sanxing Township, Yilan County 26646, Taiwan \\ ${ }^{2}$ Department of Mathematical Sciences, National ChengChi University, Taipei 11605, Taiwan
}

Correspondence should be addressed to Sheng-Ping Wang, spwang@alumni.nccu.edu.tw

Received 19 May 2010; Revised 15 September 2010; Accepted 24 September 2010

Academic Editor: Daniel Franco

Copyright (C) 2011 S.-P. Wang and L.-Y. Tsai. This is an open access article distributed under the Creative Commons Attribution License, which permits unrestricted use, distribution, and reproduction in any medium, provided the original work is properly cited.

We establish existence results of the following three-point boundary value problems: $u^{\prime \prime}(t)+$ $f(t, u(t))=0, t \in(0,1),(B C) u(0)=0$ and $u(1)=\delta u(\eta)$, where $0<\eta<1$ and $0<\delta \leq 1$. The approach applied in this paper is upper and lower solution method associated with basic degree theory or Schauder's fixed point theorem. We deal with this problem with the function $f$ which is Carathéodory or singular on its domain.

\section{Introduction}

In this paper, we consider three-point boundary value problem

$$
\begin{gathered}
u^{\prime \prime}(t)+f(t, u(t))=0, \quad t \in(0,1), \\
u(0)=0, \quad u(1)=\delta u(\eta),
\end{gathered}
$$

where $0<\eta<1$ and $0<\delta \leq 1$.

In the mathematical literature, a number of works have appeared on nonlocal boundary value problems, and one of the first of these was [1]. Il'in and Moiseev initiated the research of multipoint boundary value problems for second-order linear ordinary differential equations, see $[2,3]$, motivated by the study [4-6] of Bitsadze and Samarskii.

Recently, nonlinear multipoint boundary value problems have been receiving considerable attention, and have been studied extensively by using iteration scheme 
(e.g., [7]), fixed point theorems in cones (e.g., [8]), and the Leray-Schauder continuation theorem (e.g., [9]). We refer more detailed treatment to more interesting research [10, 11] and the references therein.

The theory of upper and lower solutions is also a powerful tool in studying boundary value problems. For the existence results of two-point boundary value problem, there already are lots of interesting works by applying this essential technique (see $[12,13])$. Recently, it is shown that this method plays an important role in proving the existence of solutions for three-point boundary value problems (see [14-16]).

Last but not least, as the singular source term appearing in two-point problems, singular three-point boundary value problems also attract more attention (e.g., [17]).

In this paper, we will discuss the existence of solutions of some general types on threepoint boundary value problems by using upper and lower solution method associated with basic degree theory or Schauder's fixed point theorem.

This paper is organized as follows. In Section 2, we give two lemmas which will be extensively used later. In Section 3, when the source term $f$ is a Carathéodory function, we consider the Sobolev space $W^{2,1}(0,1)$ defined by

$$
W^{2,1}(0,1):=\left\{u \in C^{1}[0,1] \mid u^{\prime \prime} \in L^{1}(0,1)\right\},
$$

and obtain the existence of $W^{2,1}$-solution in Theorems 3.5 and 3.11. In Section 4, we discuss the singular case, that is, $f$ maybe singular at the end points $t=0$ or $t=1$, or at $u=0$. We will introduce the $A$-class of functions and another space $W^{2, A}$ (see $[18,19]$ ) as follows:

$$
\begin{gathered}
\mathcal{A}:=\left\{h \in L_{\mathrm{loc}}^{1}(0,1) \mid s(1-s) h(s) \in L^{1}(0,1)\right\}, \\
W^{2, \mathcal{A}}(0,1):=\left\{u \in W^{1,1}(0,1) \mid u^{\prime \prime} \in \mathcal{A}\right\},
\end{gathered}
$$

and prove the existence of $W^{2, A}$-solution in Theorems 4.1 and 4.4. Some sufficient conditions for constructing upper and lower solutions are given in each section for applications.

\section{Preliminaries}

Define $G:[0,1] \times[0,1] \rightarrow(-\infty, \infty)$ by

$$
G(t, s):=\frac{1}{1-\delta \eta} t(1-s)-U(t, s)-\frac{\delta}{1-\delta \eta} V(t, s), \quad 0 \leq t, s \leq 1,
$$

where $\delta$ and $\eta$ are given as (1.2) and

$$
\begin{aligned}
& U(t, s)= \begin{cases}t-s, & s \leq t, \\
0, & t \leq s,\end{cases} \\
& V(t, s)= \begin{cases}t(\eta-s), & s \leq \eta, \\
0, & \eta \leq s .\end{cases}
\end{aligned}
$$

By direct computations, we get the following results. 
Lemma 2.1. (i) The function $G:[0,1] \times[0,1] \rightarrow(-\infty, \infty)$ defined by $(2.1)$, is the Green function corresponding for the problem

$$
\begin{gathered}
u^{\prime \prime}(t)=0, \\
u(0)=0, \quad u(1)=\delta u(\eta) .
\end{gathered}
$$

(ii) The function $G:[0,1] \times[0,1] \rightarrow(-\infty, \infty)$ defined by $(2.1)$, is continuous.

(iii) In the case $0<\delta \eta<1$, we have

$$
Q^{-1}:=\max _{0 \leq t \leq 1} \int_{0}^{1} G(t, s) d s= \begin{cases}\frac{1}{8}\left(\frac{1-\delta \eta^{2}}{1-\delta \eta}\right)^{2}, & \delta \eta(2-\eta) \leq 1, \\ \frac{\delta \eta(1-\eta)}{2(1-\delta \eta)}, & \delta \eta(2-\eta) \geq 1 .\end{cases}
$$

Lemma 2.2. If $h \in \mathcal{A}$, then the problem

$$
u^{\prime \prime}(t)+h(t)=0
$$

with boundary condition (1.2) has a unique solution $u \in W^{2, A}(0,1)$ such that

$$
u(t)=\int_{0}^{1} G(t, s) h(s) d s,
$$

where $G(t, s)$ is defined by $(2.1)$.

\section{Carathéodory Case}

In this section we first introduce the Carathéodory function as follows.

Definition 3.1. A function $f(t, u)$ defined on $E \subseteq[a, b] \times \mathbb{R}$ is called a Carathéodory function on $E$ if

(i) for almost every $t \in[a, b], f(t, \cdot)$ is continuous on $\mathbb{R}$;

(ii) for any $u \in \mathbb{R}$, the function $f(\cdot, u)$ is measurable on $[a, b]$;

(iii) for any $r>0$, there exists $h_{r} \in L(a, b)$ such that for any $u \in[-r, r]$ and for almost every $t \in[a, b]$ with $(t, u) \in E$, we have $|f(t, u)| \leq h_{r}(t)$.

We in this section assume that $f$ is a Carathéodory function and discuss the existence of $W^{2,1}$-solution by assuming the existence of upper and lower solutions.

\subsection{Existence of $W^{2,1}$-Solutions}

We first introduce the definitions of $W^{2,1}$-upper and lower solutions as below. 
Definition 3.2. A function $\alpha \in C[0,1]$ is called a $W^{2,1}$-lower solution of problem (1.1) and (1.2) if it satisfies

(i) $\alpha(0) \leq 0, \alpha(1) \leq \delta \alpha(\eta)$, and

(ii) for any $t_{0} \in(0,1)$, either $D^{-} \alpha\left(t_{0}\right)<D_{+} \alpha\left(t_{0}\right)$, or there exists an open interval $I_{0} \subseteq$ $(0,1)$ containing $t_{0}$ such that $\alpha \in W^{2,1}\left(I_{0}\right)$ and, for almost every $t \in I_{0}$, we have

$$
\alpha^{\prime \prime}(t)+f(t, \alpha(t)) \geq 0
$$

Definition 3.3. A function $\beta \in C[0,1]$ is called a $W^{2,1}$-upper solution of problem (1.1) and (1.2) if it satisfies

(i) $\beta(0) \geq 0, \beta(1) \geq \delta \beta(\eta)$, and

(ii) for any $t_{0} \in(0,1)$, either $D_{-} \beta\left(t_{0}\right)>D^{+} \beta\left(t_{0}\right)$, or there exists an open interval $I_{0} \subseteq$ $(0,1)$ containing $t_{0}$ such that $\beta \in W^{2,1}\left(I_{0}\right)$ and, for almost every $t \in I_{0}$, we have

$$
\beta^{\prime \prime}(t)+f(t, \beta(t)) \leq 0
$$
follows:

Before proving our main results, we first consider such a modified problem given as

$$
u^{\prime \prime}(t)+f(t, \gamma(t, u(t)))+\frac{\gamma(t, u(t))-u(t)}{1+|u(t)|}=0, \quad t \in(0,1)
$$

with boundary condition (1.2), where $\gamma:[0,1] \times \mathbb{R} \rightarrow \mathbb{R}$ is defined by

$$
\gamma(t, u)= \begin{cases}\alpha(t) & \text { if } u<\alpha(t) \\ u & \text { if } \alpha(t) \leq u \leq \beta(t) \\ \beta(t) & \text { if } u>\beta(t) .\end{cases}
$$

Proposition 3.4. Let $\alpha(t)$ and $\beta(t)$ be respective $W^{2,1}$-lower and upper solutions of problem (1.1) and (1.2) with $\alpha(t) \leq \beta(t)$ on $[0,1]$. If $u \in W^{2,1}(0,1)$ is a solution of problem (3.3) and (1.2), then $\alpha(t) \leq u(t) \leq \beta(t)$, for any $t \in[0,1]$.

Proof. Suppose there exists $t_{0} \in[0,1]$ such that

$$
\min _{t \in[0,1]}(u(t)-\alpha(t))=u\left(t_{0}\right)-\alpha\left(t_{0}\right)<0 .
$$

Case 1. If $t_{0} \in(0,1)$, we have $u^{\prime}\left(t_{0}\right)-D_{-} \alpha\left(t_{0}\right) \leq u^{\prime}\left(t_{0}\right)-D^{+} \alpha\left(t_{0}\right)$, which implies $D^{-} \alpha\left(t_{0}\right) \geq$ $D_{+} \alpha\left(t_{0}\right)$. Hence, by Definition 3.2 and the continuity of $u-\alpha$ at $t_{0}$, there exist an open interval $I_{0} \subseteq(0,1)$ with $t_{0} \in I_{0}, \alpha \in W^{2,1}\left(I_{0}\right)$ and a neighborhood $N$ of $t_{0}$ contained in $I_{0}$ such that for almost every $t \in I_{0} \cap N$,

$$
\alpha^{\prime \prime}(t)+f(t, \alpha(t)) \geq 0 .
$$


Furthermore, it follows from $u^{\prime}\left(t_{0}\right)-\alpha^{\prime}\left(t_{0}\right)=0$ that for $t \geq t_{0}, t \in N$, we have

$$
\begin{aligned}
u^{\prime}(t)-\alpha^{\prime}(t) & =\int_{t_{0}}^{t}\left(u^{\prime \prime}(s)-\alpha^{\prime \prime}(s)\right) d s \\
& \leq \int_{t_{0}}^{t}\left[-f(s, \gamma(s, u(s)))-\frac{\gamma(s, u(s))-u(s)}{1+|u(s)|}+f(s, \alpha(s))\right] d s \\
& =\int_{t_{0}}^{t}\left[-f(s, \alpha(s))-\frac{\alpha(s)-u(s)}{1+|u(s)|}+f(s, \alpha(s))\right] d s \\
& <0 .
\end{aligned}
$$

This implies that the minimum of $u-\alpha$ cannot occur at $t_{0}$, a contradiction.

Case 2. If $t_{0}=0$, by the definition of $W^{2,1}$-lower solution $\alpha(0) \leq 0$, we then have

$$
0=u(0) \leq u(0)-\alpha(0)<0
$$

And we get a contradiction.

Case 3. If $t_{0}=1$, it follows from the conclusion of Case 1 that

$$
u(1)-\alpha(1) \geq \delta(u(\eta)-\alpha(\eta))>\delta(u(1)-\alpha(1)) \geq u(1)-\alpha(1),
$$

which is impossible. also have

Consequently, we obtain $\alpha(t) \leq u(t)$ on $[0,1]$. By the similar arguments as above, we

$$
u(t) \leq \beta(t), \quad \text { on }[0,1]
$$

Theorem 3.5. Let $\alpha(t)$ and $\beta(t)$ be $W^{2,1}$-lower and upper solutions of problem (1.1) and (1.2) such that $\alpha(t) \leq \beta(t)$ on $[0,1]$ and let $f$ be a Carathéodory function on $E$, where

$$
E:=\{(t, u) \in[0,1] \times \mathbb{R} \mid \alpha(t) \leq u \leq \beta(t), t \in[0,1]\}
$$

Then problem (1.1) and (1.2) has at least one solution $u \in W^{2,1}(0,1)$ such that, for all $t \in[0,1]$,

$$
\alpha(t) \leq u(t) \leq \beta(t)
$$


Proof. We consider the modified problem (3.3) and (1.2) with respect to the given $\alpha(t)$ and $\beta(t)$. Consider the Banach space $C[0,1]$ with supremum and the operator $T: C[0,1] \rightarrow$ $C[0,1]$ by

$$
(T u)(t):=\int_{0}^{1} G(t, s)\left[f(s, \gamma(s, u(s)))+\frac{\gamma(s, u(s))-u(s)}{1+|u(s)|}\right] d s,
$$

for $u \in C[0,1]$, where $G(t, s)$ is defined as (2.1). Since $f$ is a Carathéodory function on $E$, for almost all $t \in[0,1]$ and for all $x \in[\alpha(t), \beta(t)]$, there exists a function $h \in L(0,1)$, we have

$$
|f(t, u)| \leq h(t)
$$

Define

$$
K:=\{u \in C[0,1] \mid\|u\| \leq M\},
$$

where

$$
\begin{gathered}
M:=\max _{t \in[0,1]} \int_{0}^{1}|G(t, s)|\left[h(s)+M_{1}\right] d s<\infty, \\
M_{1}:=\max _{(t, u) \in[0,1] \times \mathbb{R}} \frac{\gamma(t, u)-u}{1+|u|} .
\end{gathered}
$$

It is clear that $K$ is a closed, bounded and convex set in $C[0,1]$ and one can show that $T: K \rightarrow$ $K$ is a completely continuous mapping by Arzelà-Ascoli theorem and Lebesgue dominated convergence theorem. By applying Schauder's fixed point theorem, we obtain that $T$ has a fixed point in $K$ which is a solution of problem (3.3) and (1.2). From Proposition 3.4, this fixed point is also a solution of problem (1.1) and (1.2). Hence, we complete the proof. equation:

We further illustrate the use of Theorem 3.11 in the following second-order differential

$$
u^{\prime \prime}(t)+f(t, u(t))+h(t)=0
$$

with the boundary condition (1.2).

Corollary 3.6. Assume that $f:[0,1] \times \mathbb{R} \rightarrow \mathbb{R}$ is a Carathéodory function satisfying $f(t, u) / u$ is essentially bounded for $|u| \geq M$, where $M$ is a constant large enough. Assume further that $h \in L(0,1)$ and there exists a constant $0<\sqrt{A}<\pi / 2$ such that

$$
\limsup _{|u| \rightarrow \infty} \max _{t \in[0,1]} \frac{f(t, u)}{u} \leq A .
$$

Then, problem (3.18) and (1.2) has at least one solution. 
Proof. By hypothesis, for any given $\epsilon>0$ small enough such that $\sqrt{A+\epsilon} \leq \pi / 2$ and for almost all $t \in[0,1]$, for any $u$ large enough, we have

$$
f(t, u) \leq(A+\epsilon) u
$$

We now choose an upper solution $\beta(t)$ of the form

$$
\beta(t)=w(t)+s \psi(t) \geq 0
$$

To this end, we compute

$$
\begin{aligned}
\beta^{\prime \prime}+f(t, \beta)+h(t) & \leq \beta^{\prime \prime}+(A+\epsilon) \beta+h(t) \\
& =w^{\prime \prime}+(A+\epsilon) w+h(t)+s\left[\psi^{\prime \prime}+(A+\epsilon) \psi\right]
\end{aligned}
$$

Clearly, one can choose $w$ such that

$$
\begin{gathered}
w^{\prime \prime}+(A+\epsilon) w+h(t)=0, \\
w(0)=0, \quad w(1)=\delta w(\eta),
\end{gathered}
$$

that is,

$$
\begin{aligned}
w(t)= & \frac{\sin (\sqrt{A+\epsilon} t)}{\delta \sin (\sqrt{A+\epsilon} \eta)-\sin (\sqrt{A+\epsilon})} \int_{0}^{1} \frac{\sin (\sqrt{A+\epsilon}(s-1))}{\sqrt{A+\epsilon}} h(s) d s \\
& +\frac{\delta \sin (\sqrt{A+\epsilon} t)}{\delta \sin (\sqrt{A+\epsilon} \eta)-\sin (\sqrt{A+\epsilon})} \int_{0}^{\eta} \frac{\sin (\sqrt{A+\epsilon}(\eta-s))}{\sqrt{A+\epsilon}} h(s) d s \\
& +\int_{0}^{t} \frac{\sin (\sqrt{A+\epsilon}(s-t))}{\sqrt{A+\epsilon}} h(s) d s,
\end{aligned}
$$

and choose $\psi(t)=l \sin \sqrt{A+\epsilon} t$, where $l>0$, which is a positive solution of

$$
\begin{gathered}
\psi^{\prime \prime}+(A+\epsilon) \psi=0, \\
\psi(0)=0 .
\end{gathered}
$$

Hence, if $s$ is large enough, we can show that $\beta(0)=0$ and $\beta(1) \geq \delta \beta(\eta)$, where $\delta \leq 1$, which implies that $\beta(t)$ is a positive $W^{2,1}$-upper solution. In the same way we construct a $W^{2,1}$-lower solution $\alpha=w(t)-s \psi \leq 0$ on $[0,1]$. 


\subsection{Nontangency Solution}

In this subsection, we afford another stronger lower and upper solutions to get a strict inequality of the solution between them.

Definition 3.7. A function $\alpha \in C[0,1]$ is a strict $W^{2,1}$-lower solution of problem (1.1) and (1.2), if it is not a solution of problem (1.1) and (1.2), $\alpha(0)<0, \alpha(1) \leq \delta \alpha(\eta)$ and for any $t_{0} \in(0,1)$, one of the following is satisfied:

(i) $D^{-} \alpha\left(t_{0}\right)<D_{+} \alpha\left(t_{0}\right)$;

(ii) there exist an interval $I_{0} \subseteq[0,1]$ and $\epsilon>0$ such that $t_{0} \in \operatorname{int}\left(I_{0}\right), \alpha \in W^{2,1}\left(I_{0}\right)$ and for almost every $t \in I_{0}$, for all $u \in[\alpha(t), \alpha(t)+\epsilon]$ we have

$$
\alpha^{\prime \prime}(t)+f(t, u) \geq 0 .
$$

Definition 3.8. A function $\beta \in C[0,1]$ is a strict $W^{2,1}$-upper solution of problem (1.1) and (1.2), if it is not a solution of problem (1.1) and (1.2), $\beta(0)>0, \beta(1) \geq \delta \beta(\eta)$ and for any $t_{0} \in(0,1)$, one of the following is satisfied:

(i) $D_{-} \beta\left(t_{0}\right)>D^{+} \beta\left(t_{0}\right)$,

(ii) there exist an interval $I_{0} \subseteq[0,1]$ and $\epsilon>0$ such that $t_{0} \in \operatorname{int}\left(I_{0}\right), \beta \in W^{2,1}\left(I_{0}\right)$ and for almost every $t \in I_{0}$, for all $u \in[\beta(t)-\epsilon, \beta(t)]$ we have

$$
\beta^{\prime \prime}(t)+f(t, u) \leq 0
$$

Remark 3.9. Every strict $W^{2,1}$-lower(upper) solution of problem (1.1) and (1.2) is a $W^{2,1}$ lower(upper) solution.

Now we are going to show that the solution curve of problem (1.1) and (1.2) cannot be tangent to upper or lower solutions from below or above.

Proposition 3.10. Let $\alpha(t)$ and $\beta(t)$ be respective strict $W^{2,1}$-lower and upper solutions of problem (1.1) and (1.2) with $\alpha(t) \leq \beta(t)$ on $[0,1]$. If $u \in W^{2,1}(0,1)$ is a solution of problem (1.1) and (1.2) with $\alpha \leq u \leq \beta$ on $[0,1]$, then $\alpha(t)<u(t)<\beta(t)$, for any $t \in[0,1]$.

Proof. As $\alpha$ is not a solution, $u$ is not identical to $\alpha$. Assume, the conclusion does not hold, then

$$
t_{0}:=\inf \{t \in[0,1] \mid u(t)=\alpha(t)\}
$$

exists. Hence, $u-\alpha$ has minimum at $t_{0}$, that is, $u\left(t_{0}\right)-\alpha\left(t_{0}\right)=0$.

Case 1. Set $t_{0} \in(0,1)$. Since $u-\alpha$ has minimum at $t_{0}$, we have $D_{-} \alpha\left(t_{0}\right) \geq D^{+} \alpha\left(t_{0}\right)$. According to the Definition 3.7, there exist $I_{0}, \epsilon_{0}>0$ and $t_{1} \in I_{0}$ with $t_{1}<t_{0}$ such that, for every $t \in\left(t_{1}, t_{0}\right)$, $u(t) \leq \alpha(t)+\epsilon_{0}, u^{\prime}\left(t_{1}\right)-\alpha^{\prime}\left(t_{1}\right)<0$ and for a.e. $t \in\left(t_{1}, t_{0}\right)$

$$
\alpha^{\prime \prime}(t)+f(t, u(t)) \geq 0
$$


Hence, we have the contradiction since

$$
0<\left(u^{\prime}-\alpha^{\prime}\right)\left(t_{0}\right)-\left(u^{\prime}-\alpha^{\prime}\right)\left(t_{1}\right)=-\int_{t_{1}}^{t_{0}}\left[f(t, u(t))+\alpha^{\prime \prime}(t)\right] d t \leq 0 .
$$

Case 2. If $t_{0}=0$, by the definition of strict $W^{2,1}$-lower solution that $\alpha(0)<0$, we then have

$$
0=u(0)-\alpha(0)>0
$$

And we get a contradiction.

Case 3. If $t_{0}=1$, repeat the same arguments in Case 3 of the proof of Proposition 3.4. Therefore, we obtain $\alpha(t)<u(t)$ on $[0,1]$. The inequality $u(t)<\beta(t)$ on $[0,1]$ can be proved by the similar arguments as above.

Theorem 3.11. Let $\alpha(t)$ and $\beta(t)$ be strict $W^{2,1}$-lower and upper solutions of problem (1.1) and (1.2) such that $\alpha(t)<\beta(t)$ on $[0,1]$ and let $f: E \rightarrow \mathbb{R}$ be a Carathéodory function, where

$$
E:=\{(t, u) \in[0,1] \times \mathbb{R} \mid \alpha(t) \leq u(t) \leq \beta(t), t \in[0,1]\} .
$$

Then, problem (1.1) and (1.2) has at least one solution $u \in W^{2,1}(0,1)$ such that, for any $t \in[0,1]$,

$$
\alpha(t)<u(t)<\beta(t)
$$

Proof. This is a consequence of Theorem 3.5 and Proposition 3.10 and hence, we omits this proof.

\section{Singular Case}

In this section we give a more general existence result than Theorem 3.11 by assuming the existence of $W^{2,1}$-lower and upper solutions. This makes us to deal with problem (1.1) and (1.2), where the function $f$ is singular at the end point $t=0$ and $t=1$.

Theorem 4.1. Let $\alpha(t)$ and $\beta(t)$ be $W^{2,1}$-lower and upper solutions of problem (1.1) and (1.2) such that $\alpha(t) \leq \beta(t)$ on $[0,1]$ and let $f:(0,1) \times \mathbb{R} \rightarrow \mathbb{R}$ satisfy the following conditions:

(i) for almost every $t \in(0,1), f(t, \cdot)$ is continuous on $\mathbb{R}$;

(ii) for any $u \in \mathbb{R}$, the function $f(\cdot, u)$ is measurable on $(0,1)$;

(iii) there exists a function $h \in \mathcal{A}$ such that, for all $(t, u) \in E$,

$$
|f(t, u)| \leq h(t),
$$

where

$$
E:=\{(t, u) \mid t \in(0,1), \alpha(t) \leq u(t) \leq \beta(t)\} \subseteq(0,1) \times \mathbb{R}
$$


Then problem (1.1) and (1.2) has at least one solution $u \in W^{2, A}(0,1)$ such that, for all $t \in[0,1]$,

$$
\alpha(t) \leq u(t) \leq \beta(t)
$$

Proof. Consider the modified problem (3.3) and (1.2) with respect to the given $\alpha(t)$ and $\beta(t)$ and define $T: C[0,1] \rightarrow C[0,1]$ by (3.13). Note that by Lemma 2.2, $T$ is well defined. Define

$$
P:=\{u \in C[0,1] \mid\|u\| \leq N\},
$$

where

$$
N:=\max _{t \in[0,1]} \int_{0}^{1}|G(t, s)|\left[h(s)+M_{1}\right] d s<\infty,
$$

and $M_{1}$ is defined by (3.17). The rest arguments are similar to the proof of Theorem 3.5.

Remark 4.2. We have similar results of Theorems 3.5-4.1, respectively, for (1.1) equipped with

$$
u(0)=A, \quad u(1)=\delta u(\eta)
$$

where $A \in \mathbb{R}$ is a constant and $\delta, \eta$ are given as (1.2).

Example 4.3. Consider the problem (4.7), for $0<\alpha<1,0<\beta_{i}<2-2 \alpha, i=1,2$,

$$
\begin{gathered}
u^{\prime \prime}(t)+\frac{1}{t^{\beta_{1}}(1-t)^{\beta_{2}}} u(t)^{\alpha}+1=0, \quad 0<t<1, \\
u(0)=0, \quad u\left(\frac{1}{2}\right)=u(1) .
\end{gathered}
$$

Clearly, 0 is a $W^{2,1}$-lower solution of (4.7) and

$$
G(t, s):=2 t(1-s)-U(t, s)-2 V(t, s), \quad 0 \leq t, s \leq 1,
$$

where

$$
\begin{aligned}
& U(t, s)= \begin{cases}t-s, & s \leq t, \\
0, & t \leq s,\end{cases} \\
& V(t, s)= \begin{cases}t\left(\frac{1}{2}-s\right), & s \leq \frac{1}{2} \\
0, & \frac{1}{2} \leq s .\end{cases}
\end{aligned}
$$


From Lemma 2.1, we have $\max _{0 \leq t \leq 1} \int_{0}^{1} G(t, s) d s=9 / 32$ and define $h_{1}(t):=1 / t^{\beta_{1} /(1-\alpha)}(1-$ $t)^{\beta_{2} /(1-\alpha)}$. Since, for $2-\beta_{i} /(1-\alpha)>0, i=1,2$,

$$
\int_{0}^{1} t(1-t) h_{1}(t) d t=\int_{0}^{1} t^{\left(2-\beta_{1} /(1-\alpha)\right)-1}(1-t)^{\left(2-\beta_{2} /(1-\alpha)\right)-1}<\infty,
$$

that is, $h_{1}(t) \in \mathcal{A}$, we have, from Lemma 2.2, $\int_{0}^{1} G(t, s)(1-\alpha) h_{1}(s) d s \in W^{2, \mathcal{A}}$ and $\max _{0 \leq t \leq 1} \int_{0}^{1} G(t, s)(1-\alpha) h_{1}(s) d s$ exists. Let

$$
B:=\frac{1}{1-(9 / 32) \alpha} \max _{0 \leq t \leq 1} \int_{0}^{1} G(t, s)(1-\alpha) h_{1}(s) d s+1
$$

and, by Lemma 2.2 again, choose $\beta$ such that

$$
\begin{gathered}
\beta^{\prime \prime}(t)+\alpha B+(1-\alpha) h_{1}(t)+1=0, \\
\beta(0)=0, \quad \beta(1)=\beta\left(\frac{1}{2}\right) .
\end{gathered}
$$

Note that according to the direct computation, we see that $\beta$ is well-defined and is bounded by $B$. Next, let $f(t, u):=\left(1 / t^{\beta_{1}}(1-t)^{\beta_{2}}\right) u^{\alpha}+1$. By Young's inequality, it follows that

$$
\begin{aligned}
\beta^{\prime \prime}(t)+f(t, \beta(t)) & \leq \beta^{\prime \prime}(t)+\alpha \beta(t)+(1-\alpha) \frac{1}{t^{\beta_{1} /(1-\alpha)}(1-t)^{\beta_{2} /(1-\alpha)}}+1 \\
& =\beta^{\prime \prime}(t)+\alpha \beta(t)+(1-\alpha) h_{1}(t)+1 \\
& \leq 0 .
\end{aligned}
$$

Hence, such $\beta(t)$ is a $W^{2,1}$-upper solution of $(4.7)$ and $\beta(t) \geq 0$ on $[0,1]$. Clearly, $f$ satisfies (i), (ii) of Theorem 4.1. By using Young's inequality again, for $(t, u) \in E:=\{(t, u) \mid t \in(0,1), 0 \leq$ $u(t) \leq \beta(t)\} \subseteq(0,1) \times \mathbb{R}$., we have

$$
\begin{aligned}
f(t, u) & \leq \alpha u+h_{1}(t) \\
& \leq \alpha B+h_{1}(t):=h_{2}(t) .
\end{aligned}
$$

and $h_{2}(t) \in \mathcal{A}$. Therefore, $f$ satisfies the assumption (iii) of Theorem 4.1. Consequently, we conclude that this problem has at least one solution $u \in W^{2, A}(0,1)$ such that, for all $t \in[0,1]$,

$$
0 \leq u(t) \leq \beta(t)
$$

Notice that in Theorem 4.1, one can only deal with the case that $f$ is singular at end points $t=0, t=1$. However, when $f$ is singular at $u=0$, there is no hope to obtain the solutions directly from Theorem 4.1. We will establish the following theorem to deal with this case by constructing upper and lower solutions to solve this problem. 
Theorem 4.4. Assume

$\left(H_{1}\right)$ the function $f:(0,1) \times \mathbb{R}^{+} \rightarrow \mathbb{R}$ is continuous;

$\left(H_{2}\right)$ there exists $k>\pi$ and for any compact set $K \subseteq(0,1)$, there is $\epsilon>0$ such that

$$
f(t, u) \geq k^{2} u, \quad \forall t \in K, u \in(0, \epsilon]
$$

$\left(H_{3}\right)$ for some $M>0$ and $0<\gamma<\sqrt{Q}$, there is $h \in \mathcal{A} \cap C(0,1)$ such that

$$
f(t, u) \leq r^{2} u+h(t), \quad \forall t \in(0,1), u \in[M, \infty)
$$

where $Q^{-1}$ is defined as in Lemma 2.1.

$\left(H_{4}\right)$ for any compact set $K \subseteq(0, \infty)$, there is $h_{K} \in \mathcal{A}$ such that

$$
|f(t, u)| \leq h_{K}(t), \quad \forall t \in(0,1), u \in K
$$

Then problem (1.1) and (1.2) with $\delta=1$ has at least one solution

$$
u \in C\left([0,1], \mathbb{R}^{+} \cup\{0\}\right) \cap C^{2}\left((0,1), \mathbb{R}^{+}\right) .
$$

Remark 4.5 (see [12, Remark 3.1]). Assumption $\left(H_{2}\right)$ is equivalent to the assumption that there exists $k>\pi$ and a function $a_{1} \in C_{0}^{2}\left([0,1], \mathbb{R}^{+}\right)$such that:

(i) $a_{1}>0$ for all $t \in(0,1)$,

(ii) $f(t, u) \geq k^{2} u$, for all $t \in(0,1), 0<u \leq a_{1}(t)$,

(iii) $a_{1}^{\prime \prime}(t)>0$, for all $t \in[0,1 / 3] \cup[2 / 3,1]$,

where

$$
C_{0}^{2}\left([0,1], \mathbb{R}^{+}\right):=\left\{u \in C^{2}\left([0,1], \mathbb{R}^{+}\right) \mid u(0)=u(1)=0\right\}
$$

Proof.

Step 1. Construction of lower solutions. Consider $k_{2}$ such that $\pi<k_{2}<\min (k, 3 \pi)$ and the function

$$
\alpha_{2}(t)=A_{2} \cos k_{2}\left(t-\frac{1}{2}\right)
$$

where $A_{2}$ is chosen small enough so that

$$
f(t, u) \geq k^{2} u, \quad \forall t \in\left(\frac{1}{2}-\frac{\pi}{2 k_{2}}, \frac{1}{2}+\frac{\pi}{2 k_{2}}\right), 0<u \leq \alpha_{2}(t)
$$


Next, we choose $a_{1}$ from the Remark 4.5 , and let

$$
\alpha_{1}(t)=A_{1} a_{1}(t),
$$

where $A_{1} \in(0,1]$ is small enough so that for some points $t_{1} \in(0,1 / 3), t_{2} \in(2 / 3,1)$, we have:

$$
\begin{gathered}
\alpha_{1}(t) \geq \alpha_{2}(t), \quad \forall t \in\left[0, t_{1}\right] \cup\left[t_{2}, 1\right], \\
\alpha_{2}(t) \geq \alpha_{1}(t), \quad \forall t \in\left[t_{1}, t_{2}\right] .
\end{gathered}
$$

Notice that by (4.24) and (4.25), for any $h:(0,1) \times \mathbb{R}^{+} \rightarrow \mathbb{R}$ such that

$$
h(t, u) \geq f(t, u), \quad \text { for any }(t, u) \in(0,1) \times \mathbb{R}^{+},
$$

we have:

$$
\begin{gathered}
\alpha_{1}^{\prime \prime}(t)+h\left(t, \alpha_{1}(t)\right) \geq \alpha_{1}^{\prime \prime}(t)+k^{2} \alpha_{1}(t)>0, \quad \text { for any } t \in\left[0, t_{1}\right] \cup\left[t_{2}, 1\right], \\
\alpha_{2}^{\prime \prime}(t)+h\left(t, \alpha_{2}(t)\right) \geq-k_{2}^{2} \alpha_{2}(t)+k^{2} \alpha_{2}(t)>0, \quad \text { for any } t \in\left[t_{1}, t_{2}\right] .
\end{gathered}
$$

Step 2. Approximation problems. We define for each $n \in \mathbb{N}, n \geq 1$,

$$
\eta_{n}(t)=\max \left\{\frac{1}{2^{n+1}}, \min \left(t, 1-\frac{1}{2^{n+1}}\right)\right\}, \quad t \in(0,1)
$$

and set

$$
\tilde{f}_{n}(t, u)=\max \left\{f\left(\eta_{n}(t), u\right), f(t, u)\right\}
$$

We have that, for each index $n, \tilde{f}_{n}:(0,1) \times \mathbb{R}^{+} \rightarrow \mathbb{R}$ is continuous and

$$
\tilde{f}_{n}(t, u)=f(t, u), \quad \text { for any }(t, u) \in K_{n} \times \mathbb{R}^{+},
$$

where

$$
K_{n}=\left[\frac{1}{2^{n+1}}, 1-\frac{1}{2^{n+1}}\right]
$$

Hence, the sequence of functions $\left\{\tilde{f}_{n}\right\}$ converges to $f$ uniformly on any set $K \times \mathbb{R}^{+}$, where $K$ is an arbitrary compact subset of $(0,1)$. Next we define

$$
f_{n}(t, u)=\min \left\{\tilde{f}_{1}(t, u), \ldots, \tilde{f}_{n}(t, u)\right\}
$$


Each of the functions $f_{i}$ is a continuous function defined on $(0,1) \times \mathbb{R}^{+}$, moreover

$$
f_{1}(t, u) \geq f_{2}(t, u) \geq \cdots \geq f_{n}(t, u) \geq f_{n+1}(t, u) \geq \cdots \geq f(t, u)
$$

and the sequence $\left\{f_{n}\right\}$ converges to $f$ uniformly on the compact subsets of $(0,1) \times \mathbb{R}^{+}$since

$$
f_{n}(t, u)=f(t, u), \quad \forall t \in K_{n}, u \in \mathbb{R}^{+}
$$

Define now a decreasing sequence $\left\{\epsilon_{n}\right\} \subseteq \mathbb{R}^{+}$such that

$$
\begin{gathered}
\lim _{n \rightarrow \infty} \epsilon_{n}=0, \\
f(t, u) \geq k^{2} u, \quad \forall t \in K_{n}, u \in\left(0, \epsilon_{n}\right],
\end{gathered}
$$

and consider a sequence of the following approximation problems:

$$
\begin{gathered}
u^{\prime \prime}(t)+f_{n}(t, u(t))=0, \\
(B C) u(0)=\epsilon_{n}, u(1)=u(\eta),
\end{gathered}
$$

where $0<\eta<1$.

Step 3. A lower solution of $\left(P_{n}\right)$. It is clear that for any $c \in\left(0, \epsilon_{n}\right]$,

$$
\tilde{f}_{n}(t, c) \geq f\left(\eta_{n}(t), c\right) \geq k^{2} c>0
$$

As the sequence $\left\{\epsilon_{n}\right\}$ is decreasing, we also have

$$
f_{n}\left(t, \epsilon_{n}\right)=\min _{1 \leq k \leq n} \tilde{f}_{k}\left(t, \epsilon_{n}\right) \geq k^{2} \epsilon_{n}>0 .
$$

Clearly, $\alpha_{3}(t):=\epsilon_{n}$ satisfies

$$
\alpha_{3}^{\prime \prime}(t)+f_{n}\left(t, \alpha_{3}(t)\right)=f_{n}\left(t, \epsilon_{n}\right)>0 .
$$

It follows from (4.25) and (4.27) that $\alpha(t):=\max \left(\alpha_{1}(t), \alpha_{2}(t), \epsilon_{n}\right)$ is a lower solution of $\left(P_{n}\right)$. Step 4. Existence of a solution $u_{1}$ of (4.7) such that

$$
\max \left(\alpha_{1}(t), \alpha_{2}(t), \epsilon_{1}\right) \leq u_{1}(t)
$$

From assumption $\left(H_{3}\right)$, we can find $M \geq \max \left(\alpha_{1}(t), \alpha_{2}(t), \epsilon_{1}\right)$ and $h \in \mathcal{A}$ such that, for all $t \in(0,1), u \in[M, \infty)$,

$$
f(t, u) \leq r^{2} u+h(t)
$$


Also, one has

$$
f\left(\eta_{1}(t), u\right) \leq r^{2} u+h\left(\eta_{1}(t)\right) \leq r^{2} u+R,
$$

where $R>0$ is a suitable constant. Hence, we obtain, for such $t$ and $u$,

$$
f_{1}(t, u)=\max \left\{f\left(\eta_{1}(t), u\right), f(t, u)\right\} \leq r^{2} u+h(t)+R .
$$

Let $C$ be a constant such that

$$
C>\frac{1}{1-r^{2} Q^{-1}}\left\{M+\max _{0 \leq t \leq 1} \int_{0}^{1} G(t, s)(h(s)+R) d s\right\} .
$$

Choose $\beta$ such that

$$
\begin{aligned}
& \beta^{\prime \prime}(t)+\gamma^{2} C+h(t)+R=0, \\
& \beta(0)=M, \quad \beta(1)=\beta(\eta),
\end{aligned}
$$

that is,

$$
\beta(t)=M+\int_{0}^{1} G(t, s)\left(r^{2} C+h(s)+R\right) d s,
$$

where $G(t, s)$ is defined by (2.1). Note that $\beta$ is well-defined and $M \leq \beta(t) \leq C$ on $[0,1]$ since $h \in \mathcal{A}$. It is easy to see that

$$
\begin{aligned}
\beta^{\prime \prime}+f_{1}(t, \beta) & \leq \beta^{\prime \prime}+\gamma^{2} \beta+h(t)+R \\
& =\gamma^{2}(\beta-C) \\
& \leq 0
\end{aligned}
$$

So by Remark 4.2, there is a solution $u_{1}$ of (4.7) such that

$$
\max \left(\alpha_{1}(t), \alpha_{2}(t), \epsilon_{1}\right) \leq u_{1}(t) \leq \beta(t)
$$

Step 5. The problem $\left(P_{n}\right)$ has at least one solution $u_{n}$ such that

$$
\max \left(\alpha_{1}(t), \alpha_{2}(t), \epsilon_{n}\right) \leq u_{n}(t) \leq u_{n-1}(t) .
$$

Notice that $u_{n-1}$ is an upper solution of $\left(P_{n}\right)$, since

$$
\begin{gathered}
0=u_{n-1}^{\prime \prime}(t)+f_{n-1}\left(t, u_{n-1}(t)\right) \geq u_{n-1}^{\prime \prime}+f_{n}\left(t, u_{n-1}(t)\right), \\
u_{n-1}(0)=\epsilon_{n-1} \geq \epsilon_{n} .
\end{gathered}
$$


Step 6. Existence of a solution. Consider the pointwise limit

$$
\tilde{u}(t)=\lim _{n \rightarrow \infty} u_{n}(t), \quad \text { on }(0,1)
$$

It is clear that, for any $n \geq 1$,

$$
\max \left(\alpha_{1}(t), \alpha_{2}(t)\right) \leq \tilde{u}(t) \leq u_{n}(t), \quad \text { for any } t \in(0,1)
$$

and therefore $\widetilde{u}(t)>0$ on $(0,1)$. Let $K \subseteq(0,1)$ be a compact interval. There is an index $n^{*}=$ $n^{*}(K)$ such that $K \subseteq K_{n}$ for all $n \geq n^{*}$ and therefore for these $n \geq n^{*}$,

$$
0=u_{n}^{\prime \prime}(t)+f_{n}\left(t, u_{n}(t)\right)=u_{n}^{\prime \prime}(t)+f\left(t, u_{n}(t)\right), \quad \forall t \in K .
$$

Moreover, we have

$$
\sup \left\{|f(t, u)| \mid t \in K, \max \left(\alpha_{1}(t), \alpha_{2}(t)\right) \leq u \leq u_{n^{*}}(t)\right\}<\infty
$$

By Arzelá-Ascoli theorem it is standard to conclude that $\tilde{u}$ is a solution of problem (1.1) and (1.2) on the interval $K$. Since $K$ is arbitrary, we find that $\tilde{u} \in C^{2}\left((0,1), \mathbb{R}^{+}\right)$and for all $t \in(0,1)$,

$$
\tilde{u}^{\prime \prime}(t)+f(t, \tilde{u}(t))=0 .
$$

Since

$$
\tilde{u}(0)=\lim _{n \rightarrow \infty} \epsilon_{n}=0,
$$

it remains only to check the continuity of $\tilde{u}$ at $t=0$. This can be deduced from the continuity of $u_{n}$ and the fact that $u_{n}(0)=\epsilon_{n} \rightarrow 0$ as $n \rightarrow \infty$.

Example 4.6. Consider the following problem $\left(P_{2}\right)$, for $\alpha>0,0<\beta_{1}, \beta_{2}<2$,

$$
\begin{gathered}
u^{\prime \prime}(t)+\frac{1}{t^{\beta_{1}}(1-t)^{\beta_{2}}(u(t))^{\alpha}}=0, \quad 0<t<1, \\
u(0)=0, \quad u\left(\frac{1}{2}\right)=u(1) .
\end{gathered}
$$

Let $f(t, u)=1 / t^{\beta_{1}}(1-t)^{\beta_{2}} u^{\alpha}$, where $(t, u) \in(0,1) \times \mathbb{R}^{+}$. Obviously, $f$ satisfies $\left(H_{1}\right)$ and $\left(H_{4}\right)$. Moreover, for any given $k>\pi$ and for any compact set $K \subseteq(0,1)$, for $\epsilon>0$ small enough, we have

$$
f(t, u) \geq \frac{1}{u^{\alpha}}=u \frac{1}{u^{\alpha+1}} \geq k^{2} u, \quad \forall t \in K, u \in(0, \epsilon]
$$


Hence, $\left(H_{2}\right)$ holds. Furthermore, for $M>0$ large enough, $0<\gamma<4 / \sqrt{3}$, we have, from Young's inequality by choosing $1<p<\min \left\{1 / \beta_{1}, 1 / \beta_{2}\right\}$ and $1 / p+1 / q=1$,

$$
\begin{aligned}
f(t, u) & \leq \frac{1}{p} t^{-\beta_{1} p}(1-t)^{-\beta_{2} p}+\frac{1}{q} u^{-\alpha q} \\
& =h_{1}(t)+\frac{1}{q} u u^{-\alpha q-1} \\
& \leq h_{1}(t)+\gamma^{2} u, \quad \forall t \in(0,1), u \in[M, \infty),
\end{aligned}
$$

where $h_{1}:=(1 / p) t^{-\beta_{1} p}(1-t)^{-\beta_{2} p} \in \mathcal{A} \cap C(0,1)$. Hence, $\left(H_{3}\right)$ holds. By Theorem $4.4,\left(P_{2}\right)$ has at least one solution

$$
u \in C\left([0,1], \mathbb{R}^{+} \cup\{0\}\right) \cap C^{2}\left((0,1), \mathbb{R}^{+}\right) .
$$

\section{References}

[1] A. V. Bitsadze and A. A. Samarskii, "On some of the simplest generalizations of linear elliptic boundary-value problems," Doklady Akademii Nauk SSSR, vol. 185, pp. 739-740, 1969.

[2] V. A. Il'in and E. I. Moiseev, "Nonlocal boundary value problem of the first kind for a Sturm-Liouville operator in its differential and finite difference aspects," Differential Equations, vol. 23, no. 7, pp. 803$810,1987$.

[3] V. A. Il'in and E. I. Moiseev, "Nonlocal boundary value problem of the second kind for a SturmLiouville operator," Differential Equations, vol. 23, no. 7, pp. 979-987, 1987.

[4] A. V. Bitsadze, "On the theory of nonlocal boundary value problems," Soviet Mathematics—Doklady, vol. 30, no. 1, pp. 8-10, 1984.

[5] A. V. Bitsadze, "On a class of conditionally solvable nonlocal boundary value problems for harmonic functions," Soviet Mathematics—Doklady, vol. 31, no. 1, pp. 91-94, 1985.

[6] A. V. Bitsadze and A. A. Samarskii, "On some simple generalizations of linear elliptic boundary problems," Soviet Mathematics—Doklady, vol. 10, no. 2, pp. 398-400, 1969.

[7] Q. Yao, "Successive iteration and positive solution for nonlinear second-order three-point boundary value problems," Computers E Mathematics with Applications, vol. 50, no. 3-4, pp. 433-444, 2005.

[8] Q. Yao, "On the positive solutions of a second-order three-point boundary value problem with Caratheodory function," Southeast Asian Bulletin of Mathematics, vol. 28, no. 3, pp. 577-585, 2004.

[9] C. P. Gupta and S. I. Trofimchuk, "A sharper condition for the solvability of a three-point second order boundary value problem," Journal of Mathematical Analysis and Applications, vol. 205, no. 2, pp. 586-597, 1997.

[10] R. Ma, "Positive solutions of a nonlinear m-point boundary value problem," Computers E Mathematics with Applications, vol. 42, no. 6-7, pp. 755-765, 2001.

[11] H. B. Thompson and C. Tisdell, "Three-point boundary value problems for second-order, ordinary, differential equations," Mathematical and Computer Modelling, vol. 34, no. 3-4, pp. 311-318, 2001.

[12] C. De Coster and P. Habets, "Upper and lower solutions in the theory of ODE boundary value problems: classical and recent results," in Nonlinear Analysis and Boundary Value Problems for Ordinary Differential Equations, F. Zanolin, Ed., vol. 371 of CISM Courses and Lectures, pp. 1-78, Springer, Vienna, Austria, 1993.

[13] H. Lü, D. O’Regan, and R. P. Agarwal, "Upper and lower solutions for the singular $p$-Laplacian with sign changing nonlinearities and nonlinear boundary data," Journal of Computational and Applied Mathematics, vol. 181, no. 2, pp. 442-466, 2005.

[14] Z. Du, C. Xue, and W. Ge, "Multiple solutions for three-point boundary value problem with nonlinear terms depending on the first order derivative," Archiv der Mathematik, vol. 84, no. 4, pp. 341-349, 2005.

[15] R. A. Khan and J. R. L. Webb, "Existence of at least three solutions of a second-order three-point boundary value problem," Nonlinear Analysis. Theory, Methods \& Applications, vol. 64, no. 6, pp. 1356 1366, 2006. 
[16] P. Minghe and S. K. Chang, "The generalized quasilinearization method for second-order threepoint boundary value problems," Nonlinear Analysis. Theory, Methods \& Applications, vol. 68, no. 9, pp. 27792790, 2008.

[17] W. B. Qu, Z. X. Zhang, and J. D. Wu, "Positive solutions to a singular second order three-point boundary value problem," Applied Mathematics and Mechanics, vol. 23, no. 7, pp. 854-866, 2002.

[18] C. De Coster and P. Habets, Two-Point Boundary Value Problems: Lower and Upper Solutions, Springer, Berlin, Germany, 1984.

[19] P. Habets and F. Zanolin, "Upper and lower solutions for a generalized Emden-Fowler equation," Journal of Mathematical Analysis and Applications, vol. 181, no. 3, pp. 684-700, 1994. 